\title{
New somatic TERT promoter variants enhance the Telomerase activity in Glioblastoma
}

\author{
Tiziana Pierini ${ }^{1}$ (10, Carlotta Nardelli ${ }^{1}$, Anair Graciela Lema Fernandez ${ }^{1}$, Valentina Pierini ${ }^{1}$, Fabrizia Pellanera ${ }^{1}$, \\ Valeria Nofrini ${ }^{1}$, Paolo Gorello', Martina Moretti ${ }^{1}$, Silvia Arniani ${ }^{1}$, Giovanni Roti ${ }^{2}$, Paolo Giovenali' \\ Marco Lupattelli ${ }^{4}$, Giulio Metro ${ }^{5}$, Carmen Molica ${ }^{5}$, Corrado Castrioto ${ }^{6}$, Rodolfo Corinaldesi ${ }^{6}$, \\ Maria Elena Laurenti ${ }^{7}$, Stefano Ascani ${ }^{7}$, Cristina Mecucci ${ }^{1^{* \dagger}}$ and Roberta La Starza ${ }^{1^{*+}}$
}

\begin{abstract}
The catalytic activity of human Telomerase Reverse Transcriptase (TERT) compensates for the loss of telomere length, eroded during each cell cycle, to ensure a correct division of stem and germinal cells. In human tumors, ectopic TERT reactivation, most frequently due to hotspot mutations in the promoter region (TERTp), i.e. c.1-124 C>T, c.1-146 C > T, confers a proliferative advantage to neoplastic cells. In gliomas, TERTp mutations (TERTp ${ }^{\text {mut }}$ ) mainly occur in oligodendroglioma and glioblastoma. We screened, for TERTp hotspot mutations, 301 adult patients with gliomas and identified heterozygous mutations in 239 cases: $94 \%$ of oligodendroglioma, $85 \%$ of glioblastoma, and $37.5 \%$ of diffuse/anaplastic astrocytoma. Besides the recurrent c.1-124 C>T and c.1-146 C > T, two cases of glioblastoma harbored novel somatic TERTp variants, which consisted of a tandem duplications of 22 nucleotides, i.e. a TERTp c.1-100_1-79dup and TERTp c.1-110_1-89, both located downstream c.1-124 C>T and c.1-146 C > T. In silico analysis predicted the formation of 119 and 108 new transcription factor's recognition sites for TERTP c.1-100_1-79dup and TERTp c.1-110_1-89, respectively. TERTp duplications (TERTP ${ }^{\text {dup }}$ ) mainly affected the binding capacity of two transcription factors' families, i.e. the members of the E-twenty-six and the Specificity Protein/Krüppel-Like Factor groups. In fact, these new TERTpdup significantly enhanced the E-twenty-six transcription factors' binding capacity, which is also typically increased by the two C.1-124 C>T/C.1-146 C>T hotspot TERTp ${ }^{\text {mut }}$. On the other hand, they were distinguished by enhanced affinity for the Krüppel proteins. The luciferase assay confirmed that TERTp dup behaved as gain-of-function mutations causing a 2,3-2,5 fold increase of TERT transcription. The present study provides new insights into TERTp mutational spectrum occurring in central nervous system tumors, with the identification of new recurrent somatic gain-of-function mutations, occurring in $0.8 \%$ of glioblastoma IDH-wildtype.
\end{abstract}

Keywords: TERT, Gliomas, Gain-of-function mutation, ETS and Krüppel transcription factors

\section{Introduction}

The abnormal reactivation of human Telomerase Reverse Transcriptase (TERT) is a common hallmark of human solid tumors. Although it may be caused by

\footnotetext{
*Correspondence: cristina.mecucci@unipg.it; roberta.lastarza@unipg.it ${ }^{\dagger}$ Cristina Mecucci, Roberta La Starza have equally contributed to this work ${ }^{1}$ Molecular Medicine Laboratory, Centro di Ricerche Emato-Oncologiche (C.R.E.O.), S. Maria della Misericordia Hospital, University of Perugia, P.le Menghini 9, 06132 Perugia, Italy
}

Full list of author information is available at the end of the article several mechanisms, i.e. methylation, mutations, rearrangements/fusions, and DNA copy number amplifications, TERT promoter (TERTp) methylation, and gain-of-function mutations are the most frequent $[2$, 28]. In particular, two recurrent hotspot mutations are respectively located at $-124\left(T E R T \mathrm{p}^{-124}\right)$ and -146 $\left(T E R T \mathrm{p}^{-146}\right)$ base pairs (bp), from the TERT ATG start site $[2,10-12,28]$. Both mutations, generated from a cytidine to thymidine dipyrimide transition $(\mathrm{C}>\mathrm{T})$, are usually heterozygous, mutually exclusive, and produce

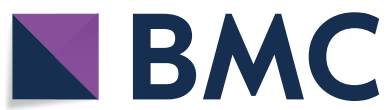

(c) The Author(s). 2020 Open Access This article is licensed under a Creative Commons Attribution 4.0 International License, which permits use, sharing, adaptation, distribution and reproduction in any medium or format, as long as you give appropriate credit to the original author(s) and the source, provide a link to the Creative Commons licence, and indicate if changes were made. The images or other third party material in this article are included in the article's Creative Commons licence, unless indicated otherwise in a credit line to the material. If material is not included in the article's Creative Commons licence and your intended use is not permitted by statutory regulation or exceeds the permitted use, you will need to obtain permission directly from the copyright holder. To view a copy of this licence, visit http://creativecommons.org/licenses/by/4.0/. The Creative Commons Public Domain Dedication waiver (http://creativeco mmons.org/publicdomain/zero/1.0/) applies to the data made available in this article, unless otherwise stated in a credit line to the data. 
an identical $11 \mathrm{bp}$ 'CCCCTTCCGGG' sequence, resulting in the creation of de novo consensus binding motifs for E-twenty-six (ETS) transcription family members. These new binding sites recruit a larger number of ETS factors, enhancing the transcription of TERT [3].

$T E R T$ promoter mutations (TERTp $\left.{ }^{\text {mut }}\right)$ typically occur in tumors that arise from low self-renewal tissue, such as melanomas, thyroid, hepatobiliary carcinoma, and central nervous system (CNS) tumors, with a variable frequency, that range from 15 to $90 \%$ of cases, in diverse histological subtypes [10, 14, 28]. In CNS tumors, TERTp $\mathrm{p}^{\text {mut }}$ are typically associated with glioblastoma (GBM) (70-80\%) and oligodendroglioma (ODG) (60-70\%), whereas their frequency decreases in other glioma subtypes, such as diffuse/ anaplastic astrocytoma (DA/AA) (30-40\%), medulloblastoma (20-30\%), and meningioma (about 7\%) $[10,25,27]$. Although the clinical value of $T E R T \mathrm{p}^{\text {mut }}$, in refining the diagnostic classification of gliomas, is widely accepted [6], its role as prognostic/predictive biomarker is still largely debated. TERTp ${ }^{\text {mut }}$ have been associated with a poor disease outcome in GBM $I D H$-wildtype (GBM $I D H^{\mathrm{wt}}$ ), but there is no full agreement on its impact on DA/AA [6, 15, 16, 22, 24, 29]. It is worth noting, however, that DA/AA $I D H$-wildtype (DA/AA $I D H^{\mathrm{wt}}$ ) harboring genomic abnormalities typically associated with GBM, i.e. TERTp mutations, or $E G F R$ amplification, or gain of whole chromosome 7 in combination with monosomy of chromosome 10, have a clinical outcome similar to, or only slightly longer, than GBM [4]. Thus, the CIMPACT NOW (Update 3) recommended to use one of these molecular criteria to classify this subgroup of astrocytomas as "diffuse astrocytic glioma, $I D H$-wildtype, with molecular features of glioblastoma, WHO grade IV" and to revise the classification of DA/AA $I D H^{\mathrm{wt}}$, accordingly [4].

Herein, we report two new TERTp mutations that were identified in two patients with GBM $I D H^{\mathrm{wt}}$. Both these new variants originated from the duplication of a stretch of 22 nucleotides at TERTp (TERTp ${ }^{\text {dup }}$ ) and, although slightly different, shared an overlapping sequence of 12 nucleotides. We demonstrated the somatic nature of one of these TERTp ${ }^{\text {dup }}$ and that, enhancing the binding affinity for ETS transcription factors (TFs), they both elicit the TERT transcription, thus widening the spectrum of recurrent gain-of-function mutations of TERTp in GBM.

\section{Case presentation Cohort}

The study was carried out on a cohort of 301 patients, affected by primary CNS tumours, and referred to our laboratory during the last 10 years (Table 1). There were
175 males and 126 females (ratio 1.4:1) with a median age of 64 (range age: 20-86). According to the WHO 2016, the diagnosis was: grade II DA $I D H^{\mathrm{wt}}$ (6 cases) and DA $I D H$-mutant (DA $\left.I D H^{\text {mut }}\right)$ (10 cases); grade III AA $I D H^{\mathrm{wt}}\left(6\right.$ cases) and AA $I D H^{\mathrm{mut}}(=10)$; grade IV GBM $I D H^{\mathrm{wt}}(=241)$ and GBM IDH ${ }^{\mathrm{mut}}(=10)$; grade II/III ODG $(=15)$. Three patients had a diagnosis of uncommon glioma (Table 1). The study was approved by Institutional Bioethics Committee (University of Perugia and Santa Maria della Misericordia Hospital of Perugia-Italy, Protocol no.2843/16); all patients gave informed consent for sample collection and molecular analyses, in agreement with the Declaration of Helsinki.

\section{Index cases}

A 71-year-old male (UPN\#131) had a left frontal lesion of $24 \mathrm{~mm}$ diameter, partially infiltrating the corpus callosum; the second case (UPN\#171), a male of 78 years, presented with a right frontal lesion. Histopathology and immunohistochemistry were consistent with a diagnosis of GBM IDH $H^{\mathrm{wt}}$, in both patients. In case UPN\#131, neoplastic cells showed marked cytoplasmic and nuclear pleomorphism; there was a discrete number of atypical mitotic figures, widespread necrosis, a diffuse GFAP positivity (100\%), and few neoplastic elements (20\%) with strong nuclear TP53 stain. Case UPN\#171, was characterized by striking atypia of neoplastic cells, diffuse necrosis, vascular proliferation, strong and diffuse positivity for GFAP and nuclear TP53 (>70\%) (Fig. 1). No IDH1/IDH2 hotspot mutations were detected, while both cases showed MGMT promoter methylation. Monosomy of chromosome 10 co-occurred with EGFR amplification (UPN\#131) or with gain of the whole chromosome 7 (UPN\#171).

\section{Materials and methods}

\section{TERT promoter mutational analysis}

Genomic DNA was extracted from Formalin-Fixed Paraffin-Embedded (FFPE) tumor tissue and from peripheral blood (PB) by QIAamp DNA FFPE and AllPrep DNA/ RNA kits, respectively, following the manufacturer's instructions (QIAGEN, Milan, Italy). Hotspot TERTpmut were investigated by Sanger sequencing using ABI 3500 Genetic analyzer instrument (Applied Biosystems, Monza, Italy). Primers were reported in Table S1 (Additional file 1: Table S1) and referred to GRCh37 genomic coordinate system (NM_000005.9, for regulatory core promoter 274 bp) (www.ncbi.nlm.nih.gov/gene [20], www.ensembl.org/Homo_sapiens [7]). Sequences' alignments and their analyses were supported by Clustal Omega (www.ebi.ac.uk/Tools/msa/clustalo), Ensembl (http://www.ensembl.org/Homo_sapiens) [7], and 
Table 1 Epidemiological and clinical features of our cohort of patients

\begin{tabular}{|c|c|c|}
\hline \multicolumn{3}{|c|}{ Epidemiological-clinical data } \\
\hline Total cohort & & 301 \\
\hline \multirow[t]{3}{*}{ Gender } & Male & 175 pts (58.1\%) \\
\hline & Female & 126 pts (41.9\%) \\
\hline & M:F & 1.4 \\
\hline \multirow[t]{4}{*}{ Age (years) } & Range & $20-86$ \\
\hline & Median & 64 \\
\hline & $<30$ years & 10 pts (3.3\%) \\
\hline & $\geq 30$ years & 291 pts $(96.7 \%)$ \\
\hline \multicolumn{3}{|l|}{ Diagnosis (WHO 2016) } \\
\hline \multirow[t]{8}{*}{ Common Gliomas } & Diffuse astrocytoma, IDH-wt (grade II) & 6 \\
\hline & Diffuse astrocytoma, IDH-mut (grade II) & 10 \\
\hline & Anaplastic astrocytoma, IDH-wt (grade III) & 6 \\
\hline & Anaplastic astrocytoma, IDH-mut (grade III) & 10 \\
\hline & Glioblastoma, IDH-wt (grade IV) & 241 \\
\hline & Glioblastoma, IDH-mut (grade IV) & 10 \\
\hline & Oligodendroglioma, IDH-mut and 1p/19q-codeleted (grade II) & 7 \\
\hline & Anaplastic oligodendroglioma, IDH-mut and 1p/19q-codeleted (grade III) & 8 \\
\hline \multirow[t]{3}{*}{ Uncommon Gliomas } & Pilocytic astrocytoma (grade I) & 1 \\
\hline & Pleomorphic xanthoastrocytoma (grade II) & 1 \\
\hline & Anaplastic pleomorphic xanthoastrocytoma (grade III) & 1 \\
\hline \multirow[t]{15}{*}{ Anatomic location } & Frontal & 97 \\
\hline & Frontal-parietal & 15 \\
\hline & Frontal-temporal & 5 \\
\hline & Parietal & 39 \\
\hline & Parietal-occipital & 10 \\
\hline & Temporal & 84 \\
\hline & Temporal-parietal & 17 \\
\hline & Temporal-occipital & 3 \\
\hline & Occipital & 9 \\
\hline & Cerebellar hemisphere & 4 \\
\hline & Corpus callosum & 2 \\
\hline & Thalamus & 1 \\
\hline & Pituitary gland & 1 \\
\hline & Insular & 1 \\
\hline & Multicentric & 13 \\
\hline
\end{tabular}

pts, patients; wt, wildtype; mut, mutant

COSMIC (https://cancer.sanger.ac.uk/cosmic) websites [5].

\section{In silico TERTp ${ }^{\text {mut }}$ functional analysis: JASPAR tool}

This bioinformatic tool estimates the binding affinity and the number of TFs binding sites for the input sequence provided in FASTA format. A relative threshold score of $80 \%$ and $\Delta$ relative score $\geq 0.05$ (mutant's relative scorewildtype's relative score) were chosen to define the statistically significant changes induced by $T E R T \mathrm{p}^{\text {mut }}$, as previously reported [1]. The JASPAR CORE predicted the effects of the four different TERTp ${ }^{\text {mut }}$ that we detected in our patients, i.e. the two new TERTp ${ }^{\text {dup }}$, the TERTp ${ }^{124}$, and the TERT $\mathrm{p}^{-146}$, on TFs binding capacity (JASPAR CORE Collection 2020; http://jaspar.genereg.net, 8th version $[8,13])$. JASPAR was also used to analyze two TERT$\mathrm{p}^{\mathrm{dup}}$, which have been previously reported in a case of MDS (c.1-110_1-101dup) and in a case of thyroid cancer (c.1-104_1-83dup) [21, 23]. According to JASPAR data, we used the Venn diagram to plot TFs for which a significant enhanced probability of binding capacity, or an 


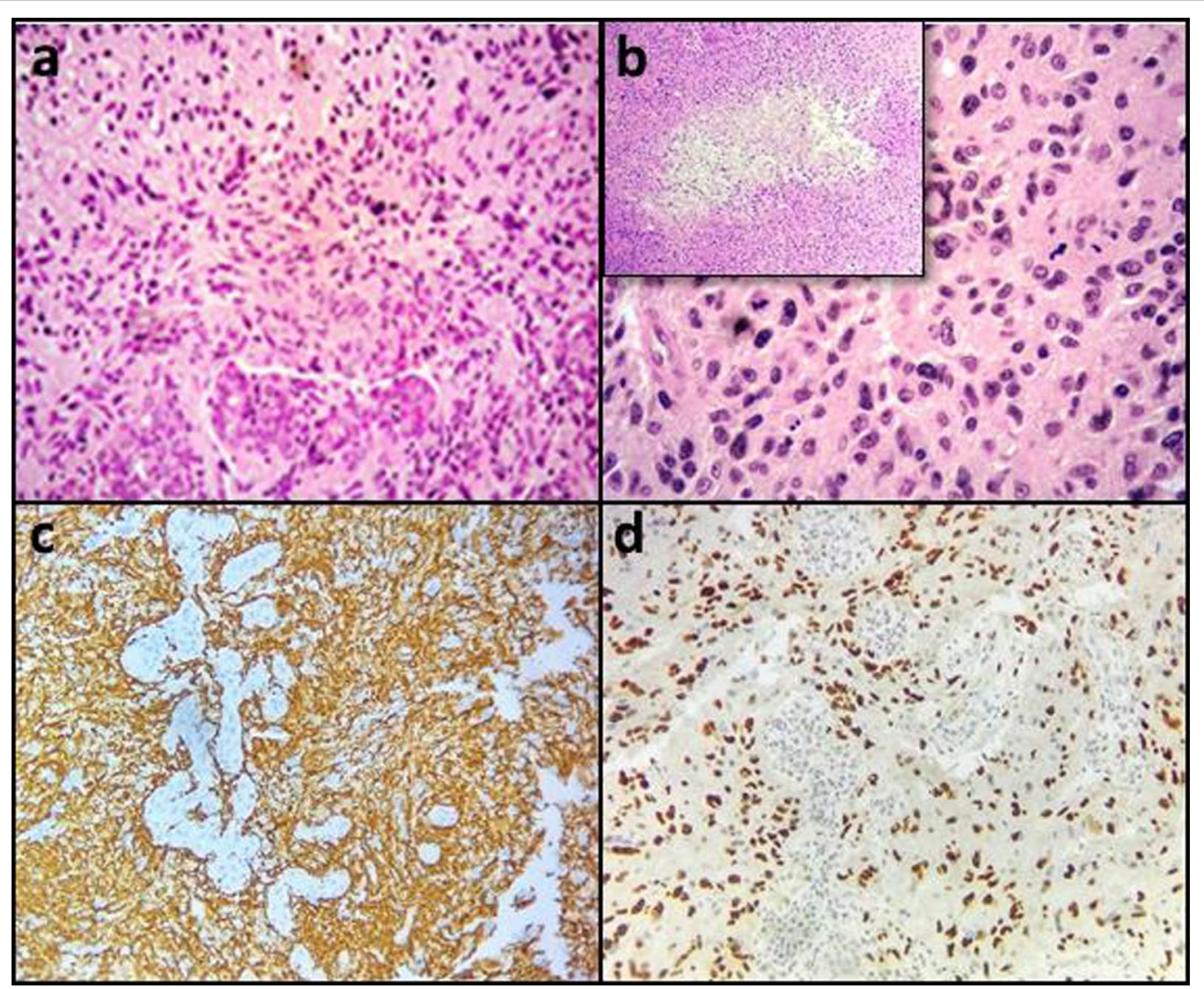

Fig. 1 Histological and immunohistochemical analysis in patient UPN\#171 a Hematoxylin/Eosin staining (original magnification 200X): enlarged neoplastic cells with multiple, often bizarre, hyperchromatic nuclei and high number of mitoses. Vascular proliferation, as seen in these "glomeruloids" (lower half of the image), is a specific pattern of microvascular growth; b Hematoxylin/Eosin staining (original magnification 400X): multiple mitotic figures are evident in the middle field. "Geographic pattern" of necrosis (detail in insert panel b); c Positive GFAP staining highlights high neoplastic cells with astrocytic differentiation; $\mathbf{d}$ Intense and diffuse nuclear TP53 staining

increase of the number of binding sites, was predicted (http://bioinformatics.psb.ugent.be/webtools/Venn/).

\section{In vitro TERTp ${ }^{\text {mut }}$ functional study: luciferase assay}

To study the effect of TERTp ${ }^{\text {mut }}$ on the expression of $T E R T$, a luciferase assay was done for the TERTp $\mathrm{p}^{\mathrm{dup}}$ detected in case UPN\#171, the TERTp ${ }^{-146}$ (UPN\#205), and the TERTp ${ }^{-124}$ (UPN\#216). The TERT dup of case UPN\#131 could not be studied due to lack of material. A TERTp wildtype $\left(T E R T \mathrm{p}^{\mathrm{wt}}\right)$ construct, already available in the laboratory, was also used as reference (Additional file 2: Table S2) [21]. TERT core promoter (310 bp) was amplified with specific primers reported in Table S3 (Additional file 3: Table S3), introducing cleavage sites for BglII (forward) and HindIII (reverse) restriction enzymes. Then, TERTp ${ }^{\text {mut }}$ constructs were inserted in pGEM-T easy plasmid (Promega, Madison WI, USA) and cloned in Electromax DH10BT1 cells (Invitrogen, Milan, Italy) to increase the amount of mutant DNA. Finally, the inserts were subcloned in pGL4.10[luc2] vectors (Promega, Madison WI, USA) upstream of $L U C 2$ gene, encoding for luciferase enzyme of Photinus Pyralis and resequenced. An empty pGL4.10[luc2] vector was also used as negative control. Luciferase assay was performed using the GBM U87-MG cell line, maintained in Dulbecco's Modified Eagle Medium (Thermo Fisher Scientific, Monza, Italy) with $10 \%$ fetal bovine serum, and $0.5 \%$ streptomycin/ penicillin at $37{ }^{\circ} \mathrm{C} / 5 \% \mathrm{CO}_{2}$. U87-MG cells were seeded in a 6 -multiwell plate $\left(3 \times 10^{5}\right.$ cells $\left./ \mathrm{ml}\right)$, co-trasfected with $3 \mu \mathrm{g}$ of modified pGL4.10[luc2] plasmids and with $1: 10$ of pGL4.74[hRluc/TK], a vector containing the luciferase gene of Renilla Reniformis, by Viafect Transfection Reagent (Promega Madison WI, USA). After 24-h incubation, cells were lysed and fluorescence emission was assessed using Dual-Glo Luciferase assay kit (Promega) following manufacturer's instructions. All experiments were performed in triplicate, in three independent experiments. 


\section{Results}

New somatic TERT promoter variants

TERT $\mathrm{p}^{\text {mut }}$ were detected in $239 / 301$ cases $(79.4 \%)$, including 14/15 ODG (93\%), 12/32 DA/AA (37.5\%), and 213/251 GBM (84.8\%) (Additional file 4: Table S4). In GBM $(=213)$ and DA/AA $(=12), T E R T \mathrm{p}^{\text {mut }}$ were prevalent in $I D H^{\mathrm{wt}}$ cases $\left(209 / 241 \mathrm{GBM}^{\mathrm{a}} I D H^{\mathrm{wt}}\right.$ vs $4 / 10 \mathrm{GBM}$ $I D H^{\text {mut }} ; 10 / 12$ DA/AA $I D H^{\text {wt }}$ vs $2 / 20$ DA/AA $I D H^{\text {mut }}$ ) (Chi square, $\mathrm{P}<0.001$ ) (Additional file 5: Table S5). Thus, in agreement with the diagnostic criteria recommended by the cIMPACT-NOW (Update 3), the $10 \mathrm{DA} / \mathrm{AA} I D H^{\mathrm{wt}}$ with TERTp ${ }^{\text {mut }}$ were referred to as "diffuse astrocytic glioma, $I D H^{-}$wildtype, with molecular features of glioblastoma, WHO grade IV" [4].

In GBM TERTp ${ }^{\text {mut }}$ there was a significant enrichment of cases harbouring EGFR amplification (46\% vs $17 \%$ ) (Chi square, $P=0.001$ ) and/or monosomy 10/PTEN deletions ( $84 \%$ vs $37.5 \%)$ (Chi square, $\mathrm{P}<0.0001$ ). Likewise, EGFR amplification or gain of whole chromosome 7 in combination with monosomy 10 , occurred in $6 / 10(60 \%)$ of TERT $\mathrm{p}^{\text {mut }} \mathrm{DA} / \mathrm{AA} I D H^{\mathrm{wt}}$.

The most common variant, TERT $\mathrm{p}^{-124}$ was detected in 172 cases while the TERTp ${ }^{-146}$ was found in 65 cases. $T E R T \mathrm{p}^{\text {mut }}$ were mutually exclusive, heterozygous, and equally distributed among the different histological subtypes (Additional file 5: Table S5). Besides the TERTp $\mathrm{p}^{-124}$ and TERTp $\mathrm{p}^{-146}$, we uncovered two new TERTp variants in two cases of GBM IDH ${ }^{\mathrm{wt}}$ (UPN\#131 and UPN\#171). These novel TERT $\mathrm{p}^{\text {mut }}$ consisted of a 22 nucleotide tandem duplication, occurring in a genomic region starting at 100 and $110 \mathrm{bp}$, from the ATG starting site, i.e. c.1100_1-79dup (TERT $\mathrm{p}^{-100-79}$ ), in case UPN\#131, and c.1110_1-89dup (TERT $\mathrm{p}^{-110-89}$ ), in case UPN\#171 (Fig. 2a, b) (www.ncbi.nlm.nih.gov/gene, www.ensembl.org/Homo_ sapiens, cancer.sanger.ac.uk/cosmic) $[5,7,20]$. They shared a region of duplication of 12 nucleotides, from $1-100$ to $1-89$ nucleotides from the ATG start site. The absence of TERT $\mathrm{p}^{-100-79}$ in the PB DNA, demonstrated the somatic origin of this variant in case UPN\#131.

\section{In silico analysis predicts TERTp ${ }^{\text {mut }}$ effects}

In silico analysis predicted that both TERT $\mathrm{p}^{\text {dup }}$ created new binding sites, i.e. 119 for TERT $\mathrm{p}^{-100-79}$ and 108 for $T E R T \mathrm{p}^{-110-89}$, which were respectively recognized by 65 and 53 TFs. Instead, TERT $\mathrm{p}^{-124}$ and TERT $\mathrm{p}^{-146}$ were predicted to increase the binding affinity for 40 and 43 sites, and to enhance the probability of binding for 28 and 29 TFs, respectively (Additional file 6: Table S6). Although all TERT $\mathrm{p}^{\text {mut }}$ affected the binding sites for diverse families of TFs, the ETS group emerged as one of the most frequently involved: $18 / 65$ (28\%) in TERTp ${ }^{-100-79}, 18 / 53$ (34\%) for TERT $\mathrm{p}^{-110-89}, 23 / 28(82 \%)$ in TERT $\mathrm{p}^{-124}$, and 25/29 (86\%) in TERT ${ }^{-146}$, (Fig. 2c, Additional file 7:
Table S7). Other recurrently involved TFs in TERT $\mathrm{p}^{\text {dup }}$ variants were the Specificity Protein/Krüppel-Like Factor (Sp/KLF) family, i.e. 19/65 (29\%) in TERT $\mathrm{p}^{-100-79}$ and $16 / 53(30 \%)$ in TERT $\mathrm{p}^{-110-89}$, and the More than 3 adjacent zinc finger factors family $\left(12 / 65\right.$ in TERT $\mathrm{p}^{-100-79}$ and 7/53 TERT $\mathrm{p}^{-110-89}$ ) (Additional file 7: Table S7).

The Venn diagram showed a close inter-relationship between all TERTp mutations. Namely, all TERTp mutations shared an increase of the binding affinity, or the number of binding motifs, for 19 common TFs (Fig. 3a), including 18 ETS members (ETS1, ETS2, ERG, ELK1, ETV6, FLI1, ELK4, SPIB, ELF1, ELF3, ETV4, ETV1, FEV, EHF, ETV5, ELF5, SPI1, and GABPA) and TEAD1 (Fig. 3a; Additional file 8: Table S8). The Venn diagram also showed that the new $T E R T \mathrm{p}^{\text {dup }}$ were characterized by the exclusive involvement of 30 common TFs. Specifically, there were $16 \mathrm{Sp} / \mathrm{KLF}$ members, i.e. KLF2, KLF3, KLF4, KLF5, KLF10, KLF11, KLF14, KLF15, KLF16, SP1, SP2, SP3, SP4, SP8, SP9, and EGR1, (Fig. 3a, Additional file 8: Table S8) and 14 TFs that belong to 9 different families (Fig. 3a, Additional files 7 and 8: Tables S7 and S8). Matching our TERT $\mathrm{p}^{\text {dup }}$ with the two cases of TERT$\mathrm{p}^{\text {dup }}$ previously reported (Additional files 9 and 10: Tables S9 and S10) [21, 23], JASPAR predicted that all variants determined an increase of binding sites for 21 common TFs, and confirmed that the Sp/KLF family was the most frequently involved (14/21) (Fig. 3b, Additional file 11: Table S11).

\section{In vitro analysis confirms the increasing of TERT} transcriptional activity induced by its promoter mutations In vitro luciferase assay was carried out to evaluate whether the new TERT $\mathrm{p}^{-110-89}$ variant induced an increase of TERT transcriptional activity, enhancing its expression, similarly to $T E R T \mathrm{p}^{-124}$ and $T E R T \mathrm{p}^{-146}$ $[12,21]$. In Table S12 (Additional file 12: Table S12) we reported raw data referred to the fluorescence emission values, expressed in Relative Luciferase Activity (RLA), of both Photinus Pyralis and Renilla Reniformis luciferase enzymes, for all samples. Our experiments demonstrated that all three variants caused a significant increase of TERT transcription by 2.3-2.5 fold than wildtype

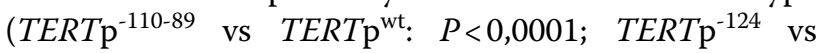

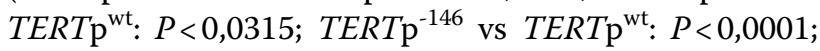
Mann-Whitney U test) (Fig. 4). On the other hand, no differences on the levels of TERT expression were present between the diverse TERTp variants, indicating they may all behave as gain-of-function mutations, likely exerting the same consequences on TERT transcription. 


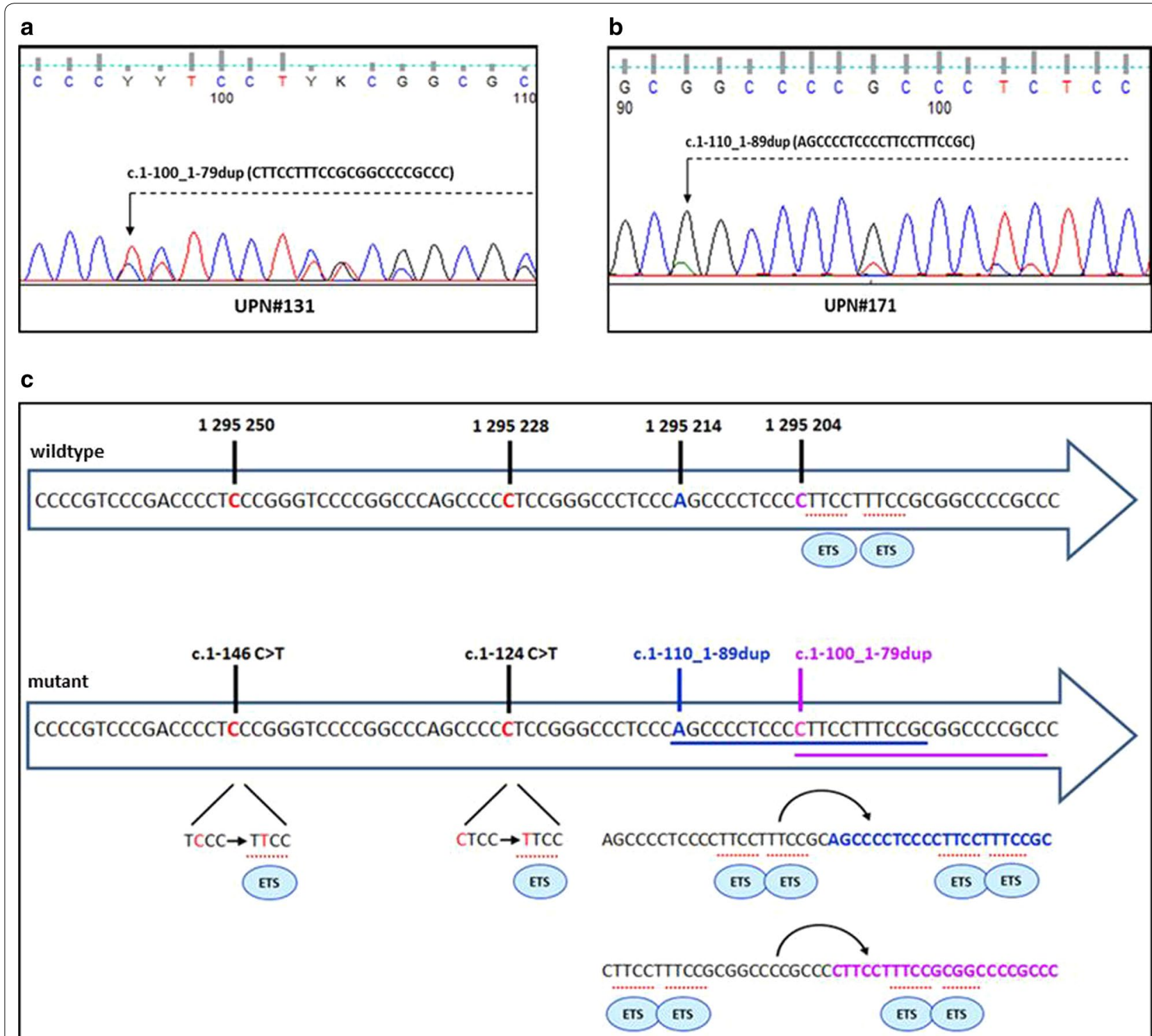

Fig. 2 Schematic representation of TERTp mutations: a TERT promoter electropherogram in case UPN\#131. The arrow indicates the start point of the c.1-100_1-79dup; b TERT promoter electropherogram in case UPN\#171. The arrow indicates the start point of the c.1-110_1-89dup; c Overview of all TERTp variants detected in our cases. Upper arrow: wildtype TERT core promoter with the normal location of ETS binding sites. The vertical black lines indicate the genomic positions of TERTp variants. Lower arrow: positions and types of TERTp variants and their predicted effects on transcription factors binding sites

\section{Discussion}

Abnormal genomic events that alter telomere elongation are common in gliomas. Particularly, mutually exclusive mutations affect the TERT or the ATRX chromatin remodeler $(A T R X)$ genes, a critical regulator of telomere homeostasis by chromatin remodeling [9].

Our studies, on a cohort of 301 patients, confirmed previous data on the incidence and distribution of TERTp ${ }^{\text {mut }}$ in diverse subtypes of CNS tumors. As expected, we found that $T E R T \mathrm{p}^{\text {mut }}$ were highly recurrent in ODG and GBM, and less frequent in DA/ AA (Additional file 4: Table S4). TERTp ${ }^{\text {mut }}$ were significantly enriched in GBM $I D H^{\mathrm{wt}}$ cases $(83 \%)$ (Chi square, $\mathrm{P}<0.001$ ) (Additional file 55: Table S5), where they mainly occurred together with EGFR amplification (Chi square, $\mathrm{P}=0.001$ ) and/or monosomy 10/PTEN deletions (Chi square, $\mathrm{P}<0.0001$ ). Similarly, in $\mathrm{DA} /$ $\mathrm{AA}, T E R T \mathrm{p}^{\text {mut }}$ were highly recurrent in $I D H^{\mathrm{wt}}$ cases, thus allowing the reclassification of $83 \%$ of these subgroup of astrocytomas as "diffuse astrocytic glioma, 


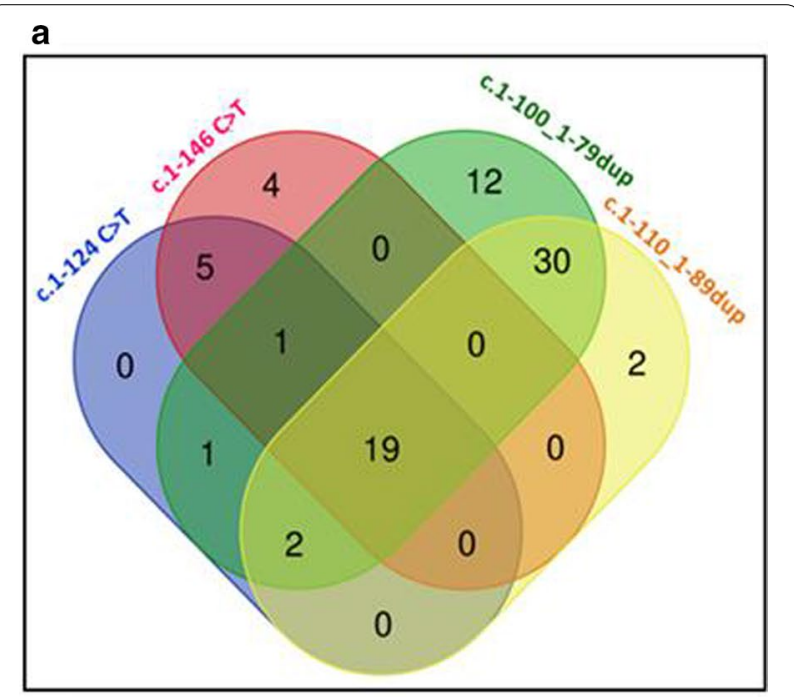

b

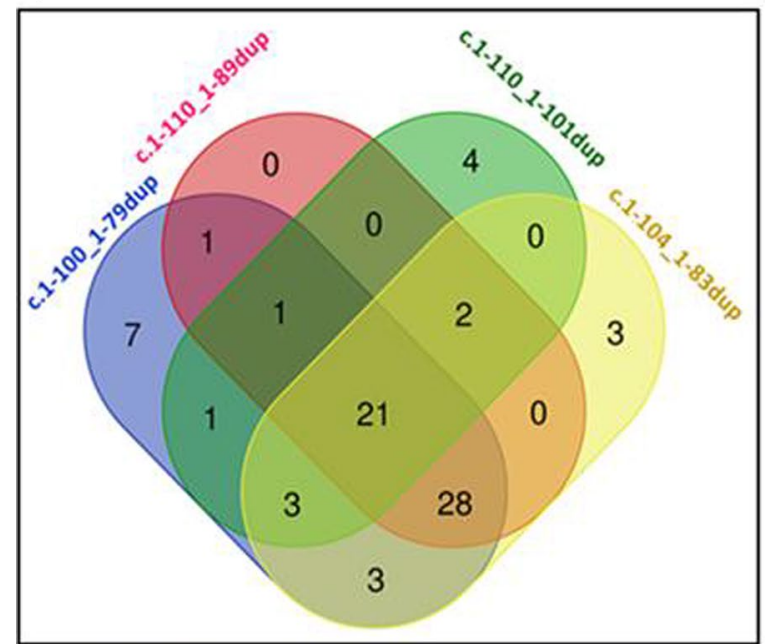

Fig. 3 The Venn diagrams show all possible relations among: a four TERTp variants reported in our cases (refer to Additional file 8: Table S8) and; $\mathbf{b}$ TERTp ${ }^{\text {dup }}$ described in this study (c.1-100_1-79dup and c.1-110_1-89dup) and those reported in literature (c.1-104_1-83dup and c.1-110_1-101dup) (refer to Additional file 11: Table S11)

$I D H$-wildtype, with molecular features of glioblastoma, WHO grade IV" [4].

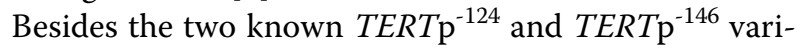
ants, we uncovered two new TERTp variants in two cases of GBM IDH ${ }^{\mathrm{wt}}$ (UPN\#131 and UPN\#171). These novel TERTp ${ }^{\text {mut }}$ consisted of a 22 nucleotide tandem duplication, sharing a duplicated region of 12 nucleotides, from 1-100 to 1-89, from the ATG start site. Hitherto, somatic TERTp ${ }^{\text {dup }}$ has been reported in three human tumors. The first one, a duplication of 41 nucleotides in the TERT core promoter, was detected in a case of
ODG [3]. Afterwards, TERTp ${ }^{\text {dup }}$ were found in a case of myelodysplastic syndrome (MDS) (c.1-110_1-101dup) and in a case of papillary thyroid carcinoma (c.1-104_183dup) [21, 23]. Published TERTp ${ }^{\text {dup }}$ as well as our cases, are located in the same core promoter region, that span 1-110/1-79 bp from the ATG start site. Furthermore, they are all located downstream TERTp ${ }^{-124}$ and TERT $\mathrm{p}^{-}$ 146, i.e. at 13-23 nucleotides from $T E R T \mathrm{p}^{-124}$ and 35-45 nucleotides from $T E R T \mathrm{p}^{-146}$, in a region that contains the binding sites for the TFs modulating TERT transcription. Interestingly, in silico analysis predicted these new $T E R T^{\text {dup }}$ affect the transcriptional regulation of the gene through the creation of new binding sites for TFs that mainly belong to the ETS family (Fig. 2c, Additional file 7: Table S7). Likewise, an increased number of binding sites or an enhanced affinity for the ETS TFs, has been previously reported in a thyroid cancer harbouring a TERTp c.1-104_1-83dup variant, and in cases bearing $T E R T \mathrm{p}^{-124}$ or TERT $\mathrm{p}^{-146}$ mutations $[3,10,23]$. Bioinformatic analyses were consistent with the luciferase data showing a significant increase of TERT expression in cells transfected with the new TERTp ${ }^{-110-89}$ variant as well as with the two recurrent $T E R T \mathrm{p}^{\text {mut }}$.

Then, we sought to assess the possible inter-relationship between the four diverse TERTp mutations using the Venn diagram (Fig. 3a). All four TERTp variants were predicted to share an increase binding capacity for 18 ETS members (Fig. 3a; Additional file 8: Table S8), which included GABPA, a putative oncogene in GBM. Namely, in vitro studies on GBM cell lines have demonstrated that this transcription factor is needful in mediating the transcriptional reactivation of TERT dependent from TERTp ${ }^{124}$ or TERTp ${ }^{-146}[3,10,19]$. Besides ETS TFs, all TERTp variants affected the binding capacity for TEAD1, a protein that belongs to TEF-1-related factors family, and that has been demonstrated to act as a putative oncogene in GBM, favoring cell infiltration in vitro/in vivo models [26].

Although TERT $\mathrm{p}^{-124}$ and $T E R T \mathrm{p}^{-146}$, and the new TERT $\mathrm{p}^{-100-79}$ and TERT $\mathrm{p}^{-110-89}$ variants, shared the same effects on the binding capacity for ETS members, the latters were characterized by the exclusive involvement of $30 \mathrm{TFs}$, mainly belonging to Sp/KLF family (Fig. 3a, Additional files 7 and 8: Tables S7 and S8). Sp/KLF TFs are involved in a plethora of cellular processes ranging from proliferation and differentiation, pluripotency and apoptosis, in normal and tumoral tissues [17].

Altogether these data support the hypothesis that the recruitment of ETS family TFs plays a pivotal role in mediating the reactivation of TERT transcription in human tumors bearing different types of TERTpmut. However, they also indicate that slight differences mark TERTp ${ }^{\text {dup }}$ variants, whose activities appear to be 


\section{TERTp mutations effect on luciferase's assay}

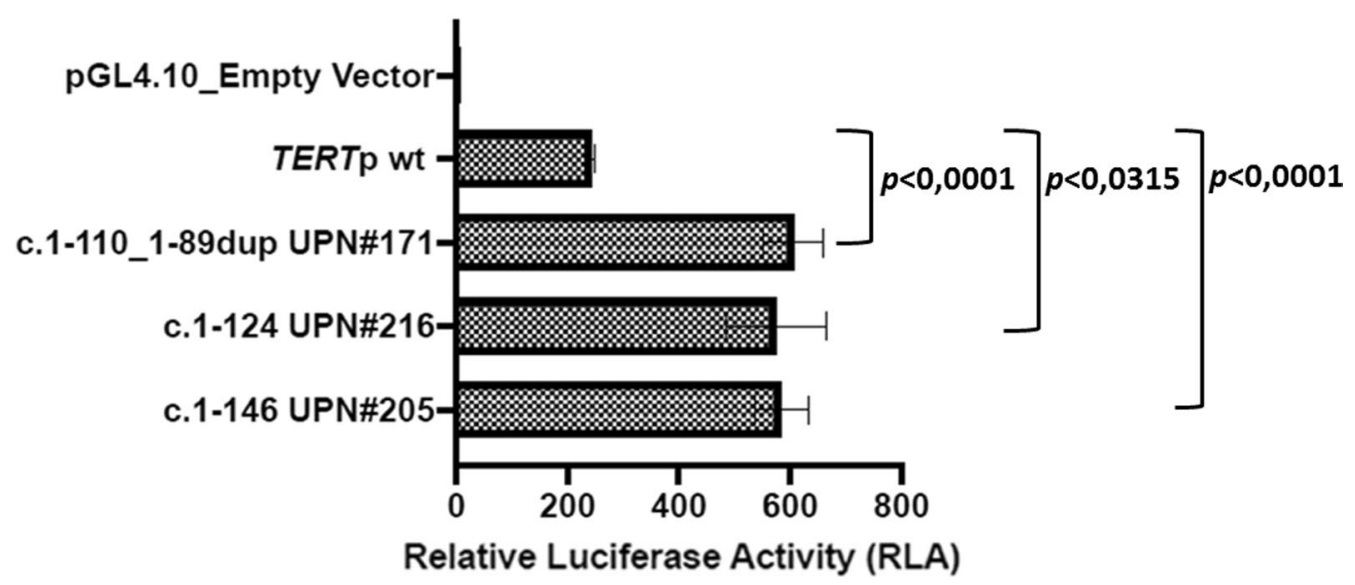

Fig. 4 Luciferase assay. The histogram reports the relative luciferase activities (RLA) of TERTp wildtype and for the variants c.1-110_1-89dup, c.1-124 C>T, and c.1-146C C T. p value refers to probability obtained using Mann-Whitney U test

also dependent from Krüppel-related factors. Indeed, among the 21 TFs shared by all TERTp ${ }^{\text {dup }}$ (Fig. 3b), 14 belonged to Sp/KLF family (67\%) as reported in Tables S10 and S11 (Additional files: 10 and 11). Hence, the precise definition of mutation-specific profiles would strengthen the definition of TERT-dependent oncogenesis mechanisms.

Our study contributes to enrich the spectrum of recurrent somatic TERT $\mathrm{p}^{\text {dup }}$ variants reporting, for the first time, two new gain-of-function mutations, i.e. TERT $\mathrm{p}^{-100-79}$ and TERT $\mathrm{p}^{-110-89}$, in $0.8 \%$ of GBM $I D H^{\mathrm{wt}}$ cases. These new mutations can be reliably detected by diagnostic assays used to investigate hotspot TERTp124 and TERT $\mathrm{p}^{-146}$. Although the assessment of TERTp mutational status is not an essential diagnostic criterion, it can be a relevant information to assist histological diagnosis [18]. As a matter of fact, the status of TERTp, together with $I D H$ mutations and $1 \mathrm{p} / 19 \mathrm{q}$ co-deletion, classify gliomas in 5 distinct subcategories, i.e. triple negative, triple positive, cases with IDH/TERT mutations, and cases with a unique mutation (either IDH or TERT), that are typified by unique demographic, clinical and biological characteristics [6]. Moreover, TERTp ${ }^{\text {mut }}$ has been proposed as one of the most relevant molecular marker to stratify DA/ AA $I D H^{\mathrm{wt}}$ [4]. Thus, we consider that molecular testing of TERTp mutations should be included in the clinical work-up of GBM and DA/AA in order to provide a precise diagnosis: prospective multicentric studies, on large cohort of patients, will clarify the value of TERTp mutations as prognostic marker.

\section{Supplementary information}

Supplementary information accompanies this paper at https://doi. org/10.1186/s40478-020-01022-4.

Additional file 1: Table S1. Primer set used for Sanger sequencing. Additional file 2: Table S2.Samples used for in vitro luciferase assay. Additional file 3: Table S3. Primer set used to create constructs for luciferase assay.

Additional file 4: Table S4. Incidence and distribution of TERTp variants in the main glioma subgroups.

Additional file 5: Table S5. Incidence and distribution of TERTp variants in glioma subtypes (according to WHO 2016 guidelines).

Additional file 6: Table S6. JASPAR analysis for the TERTP c.1-124 C>T, c.1-

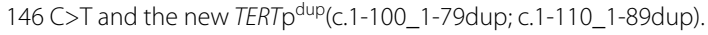

Additional file 7: Table S7. Transcription Factors predicted to be involved in TERTp variants.

Additional file 8: Table S8. Transcription Factors predicted to be involved in different TERTp variants.

Additional file 9: Table S9. JASPAR analysis for the two published TERTp duplications c.1-110_1-101dup and c.1-104_1-83dup [ref. 21, 23].

Additional file 10: Table S10. Transcription factors predicted to be involved in the TERTP ${ }^{\text {dup }}$ c.1-110_1-101dup and c.1-104_1-83dup [ref. 21, 23].

Additional file 11: Table S11. Transcription factors predicted to be involved in all TERTP duplications.

Additional file 12: Table S12. Luciferase assay: raw data.

\section{Abbreviations}

TERT: Telomerase Reverse Transcriptase; TERTp: TERT promoter; TERTp mut. TERT promoter mutation; TERTp ${ }^{\text {dup: }}$ TERT promoter duplication; TERTp ${ }^{-124}$ : c.1-124 TERT promoter mutation; TERTP ${ }^{-146}$ : c.1-146 TERT promoter mutation; bp: base pair; ETS: E-twenty-six transcription factor; CNS: central nervous system; GBM: glioblastoma; ODG: oligodendroglioma; DA: diffuse astrocytoma; AA: anaplastic astrocytoma; GBM IDH $H^{\text {wt: }}$ glioblastoma IDH-wildtype; DA IDH't: diffuse 
astrocytoma IDH-wildtype; AA IDH ${ }^{\text {wt: }}$ anaplastic astrocytoma IDH-wildtype; TFs: transcription factors; DA IDH'mut: diffuse astrocytoma IDH-mutant; AA IDH ${ }^{\text {mut: }}$ anaplastic astrocytoma IDH-mutant; GBM IDH ${ }^{\text {mut. }}$ glioblastoma IDH-mutant; FFPE: formalin-fixed paraffin-embedded; PB: peripheral blood; MDS: myelodysplastic syndrome; TERTp ${ }^{\text {wt: }}$ TERTp wildtype; TERTp ${ }^{-100-79}:$ c.1-100_1-79dup; TERTP ${ }^{-110-89}$ : c.1-110_1-89dup; Sp/KLF: Specificity Protein/Krüppel-Like Factor; RLA: relative luciferase activity; ATRX: ATRX chromatin remodeler.

\section{Acknowledgements}

Not applicable.

\section{Authors' contributions}

TP, RLS conceived the study, planned the experiments, and wrote the paper; TP carried out and evaluated mutational analysis and in vitro functional studies; CN made in silico analysis; AGLF, contributed in the analysis of in vitro luciferase assay; MM and SA performed DNA extraction and FISH experiments; $F P, V N$ and $P G$ performed sequencing analysis; $P G, S A$, and MEL provided the diagnosis and the tissue sections for molecular-cytogenetic studies; $\mathrm{CC}$ and RC, ML, GM and CM provided all clinical data; VP, GR and CM were involved in drafting the manuscript. All the authors read and approved the final manuscript.

\section{Funding}

The project was supported by Comitato per la vita "Daniele Chianelli", Perugia, Italy; Sergio Luciani Association, Fabriano, Italy; and Fondazione Cassa di Risparmio Perugia, Italy (Grant numbers: 2018.0418.021 to RLS).

\section{Availability of data and materials}

All data generated or analyzed during this study are included in this published article [and in its supplementary information files].

\section{Ethics approval and consent to participate}

This study was approved by the local ethic committee C.E.A.S. code number 2843/16, August 8th, 2016.

\section{Consent for publication}

All participants signed an institutional informed consent.

\section{Competing interests}

The authors declare that they have no competing interests.

\begin{abstract}
Author details
${ }^{1}$ Molecular Medicine Laboratory, Centro di Ricerche Emato-Oncologiche (C.R.E.O.), S. Maria della Misericordia Hospital, University of Perugia, P.le Menghini 9, 06132 Perugia, Italy. ${ }^{2}$ Hematology and Center of Bone Marrow Transplants, Medicine and Surgery Department, University and Hospital of Parma, Via Gramsci 14, 43126 Parma, Italy. ${ }^{3}$ Diagnostic Cytology and Histology Unit, S. Maria della Misericordia Hospital, P.le Giorgio Menghini 8/9, 06132 Perugia, Italy. ${ }^{4}$ Division of Radiotherapy, S. Maria della Misericordia Hospital, P.le Giorgio Menghini 8/9, 06132 Perugia, Italy. ${ }^{5}$ Medical Oncology, S. Maria della Misericordia Hospital, P.le Giorgio Menghini 8/9, 06132 Perugia, Italy. ${ }^{6}$ Division of Neurosurgery, S. Maria della Misericordia Hospital, P.le Giorgio Menghini 8/9, 06132 Perugia, Italy. ${ }^{7}$ Pathology Unit, S. Maria Hospital, V. Tristano di Joannuccio, 05100 Terni, Italy.
\end{abstract}

Received: 4 June 2020 Accepted: 12 August 2020

Published online: 25 August 2020

\section{References}

1. Allory Y, Beukers W, Sagrera A, Flández M, Marqués M, Márquez M et al (2014) Telomerase Reverse Transcriptase promoter mutations in bladder cancer: high frequency across stages, detection in urine, and lack of association with outcome. Eur Urol 65:360-366

2. Barthel FP, Wei W, Tang M, Martinez-Ledesma E, Hu X, Amin SB et al (2017) Systematic analysis of telomere length and somatic alterations in 31 cancer types. Nat Genet 49:349-357
3. Bell RJ, Rube HT, Kreig A, Mancini A, Fouse SD, Nagarajan RP et al (2015) The transcription factor GABP selectively binds and activates the mutant TERT promoter in cancer. Science 348:1036-1039

4. Brat DJ, Aldape K, Colman H, Holland EC, Louis DN, Jenkins RB et al (2018) CIMPACT-NOW update 3: recommended diagnostic criteria for "Diffuse astrocytic glioma, IDH-wildtype, with molecular features of glioblastoma, WHO grade IV". Acta Neuropathol 136:805-810

5. COSMIC, Catalogue of Somatic Mutations in Cancer Database (2020) Wellcome Sanger Institute, Cambridge UK. https://cancer.sanger.ac.uK/ cosmic. Accessed 30 May 2020

6. Eckel-Passow JE, Lachance DH, Molinaro AM, Walsh KM, Decker PA, Sicotte $\mathrm{H}$ et al (2015) Glioma groups based on 1p/19q, IDH, and TERT promoter mutations in tumors. N Engl J Med 372:2499-2508

7. Ensembl Database-Homo Sapiens (2020) European Molecular Biology Laboratory's European Bioinformatics Institute, Cambridge UK. http:// www.ensembl.org/Homo_sapiens. Accessed 30 May 2020

8. Fornes O, Castro-Mondragon JA, Khan A, van der Lee R, Zhang X, Richmond PA et al (2020) JASPAR 2020: update of the open-access database of transcription factor binding profiles. Nucleic Acids Res 48:D87-D92. https://doi.org/10.1093/nar/gkz1001

9. Haase S, Garcia-Fabiani MB, Carney S, Altshuler D, Núñez FJ, Méndez FM et al (2018) Mutant ATRX: uncovering a new therapeutic target for glioma. Expert Opin Ther Targets 22:599-613

10. Heidenreich B, Kumar R (2017) TERT promoter mutations in telomere biology. Mutat Res 771:15-31

11. Horn S, Figl A, Rachakonda PS, Fischer C, Sucker A, Gast A et al (2013) TERT promoter mutations in familial and sporadic melanoma. Science 339:959-961

12. Huang FW, Hodis E, Xu MJ, Kryukov GV, Chin L, Garraway LA (2013) Highly recurrent TERT promoter mutations in human melanoma. Science 339:957-959

13. JASPAR CORE Collection Database (2020) University of Copenhagen, Centre for Molecular Medicine and Therapeutics, London Institute of Medical Sciences, Centre for Molecular Medicine Norway. http://jaspa r.genereg.net. Accessed 30 May 2020

14. Killela PJ, Reitman ZJ, Jiao Y, Bettegowda C, Agrawal N, Diaz LA Jr et al (2013) TERT promoter mutations occur frequently in gliomas and a subset of tumors derived from cells with low rates of self-renewal. Proc Natl Acad Sci USA 110:6021-6026

15. Labussière M, Di Stefano AL, Gleize V, Boisselier B, Giry M, Mangesius S et al (2014) TERT promoter mutations in gliomas, genetic associations and clinico-pathological correlations. Br J Cancer 111:2024-2032

16. Lee Y, Koh J, Kim SI, Won JK, Park CK, Choi SH et al (2017) The frequency and prognostic effect of TERT promoter mutation in diffuse gliomas. Acta Neuropathol Commun 5:62

17. Limame R, Op de Beeck K, Lardon F, De Wever O, Pauwels P (2014) Krüppel-like factors in cancer progression: three fingers on the steering wheel. Oncotarget 5:29-48

18. Louis DN, Perry A, Reifenberger G, von Deimling A, Figarella-Branger D, Cavenee WK et al (2016) The 2016 World Health Organization Classification of tumors of the central nervous system: a summary. Acta Neuropathol 131:803-820

19. Makowski MM, Willems E, Fang J, Choi J, Zhang T, Jansen PW et al (2016) An interaction proteomics survey of transcription factor binding at recurrent TERT promoter mutations. Proteomics 16:417-426

20. National Center for Biotechnology Information Database-Gene (2019) U.S. National Library of Medicine, Rockville Pike. http://www.ncbi.nlm.nih. gov/gene. Accessed 30 May 2020

21. Nofrini V, Matteucci C, Pellanera F, Gorello P, Di Giacomo D, Lema Fernandez AG et al (2020) Activating somatic and germline TERT promoter variants in myeloid malignancies. Leukemia [Online ahead of print]

22. Nonoguchi N, Ohta T, Oh JE, Kim YH, Kleihues P, Ohgaki H (2013) TERT promoter mutations in primary and secondary glioblastomas. Acta Neuropathol 126:931-937

23. Panebianco F, Nikitski AV, Nikiforova MN, Nikiforov YE (2019) Spectrum of TERT promoter mutations and mechanisms of activation in thyroid cancer. Cancer Med 8:5831-5839

24. Pekmezci M, Rice T, Molinaro AM, Walsh KM, Decker PA, Hansen $\mathrm{H}$ et al (2017) Adult infiltrating gliomas with WHO 2016 integrated diagnosis: 
additional prognostic roles of ATRX and TERT. Acta Neuropathol 133:1001-1016

25. Sahm F, Schrimpf D, Olar A, Koelsche C, Reuss D, Bissel J et al (2015) TERT promoter mutations and risk of recurrence in meningioma. J Natl Cancer Inst 108:djv377. https://doi.org/10.1093/jnci/djv377

26. Tome-Garcia J, Erfani P, Nudelman G, Tsankov AM, Katsyv I, Tejero R et al (2018) Analysis of chromatin accessibility uncovers TEAD1 as a regulator of migration in human glioblastoma. Nat Commun 9:4020

27. Viana-Pereira M, Almeida GC, Stavale JN, Malheiro S, Clara C, Lobo P et al (2017) Study of hTERT and histone 3 mutations in medulloblastoma. Pathobiology 84:108-113

28. Yuan X, Larsson C, Xu D (2019) Mechanisms underlying the activation of TERT transcription and telomerase activity in human cancer: old actors and new players. Oncogene 38:6172-6183
29. Zhang ZY, Chan AK, Ding XJ, Qin ZY, Hong CS, Chen LC et al (2015) TERT promoter mutations contribute to IDH mutations in predicting differential responses to adjuvant therapies in WHO grade II and III diffuse gliomas. Oncotarget 6:24871-24883

\section{Publisher's Note}

Springer Nature remains neutral with regard to jurisdictional claims in published maps and institutional affiliations.
Ready to submit your research? Choose BMC and benefit from:

- fast, convenient online submission

- thorough peer review by experienced researchers in your field

- rapid publication on acceptance

- support for research data, including large and complex data types

- gold Open Access which fosters wider collaboration and increased citations

- maximum visibility for your research: over $100 \mathrm{M}$ website views per year

At BMC, research is always in progress.

Learn more biomedcentral.com/submissions 